\title{
Barotrauma developed during intra-hospital transfer -A case report-
}

\author{
Jong Bun Kim, Hyun-Ju Jung, Jae Myeong Lee, Kyong Shil Im, and Duk Joo Kim \\ Department of Anesthesiology and Pain Medicine, The Catholic University of Korea College of Medicine, Seoul, Korea
}

A 74-year-old male patient receiving ventilatory support due to aspiration pneumonia developed bilateral pneumothorax, pneumopericardium, pneumomediastinum, pneumo-retroperitoneum, and subcutaneous emphysema, after manual ventilation while being transferred from the intensive care unit (ICU) to the operating room (OR). These complications were assumed to be secondary to inappropriate manual ventilation of the intubated patient. In addition, it is likely that the possible migration of an already marginally acceptable endotracheal tube (ETT) position during transport was the cause of these complications. Finally, aggravation of a latent pneumothorax might have contributed to these complications. (Korean J Anesthesiol 2010; 59: S218-S221)

Key Words: Barotrauma, Transfer, Ventilation.

Barotrauma is a potentially lethal complication of positive pressure ventilation. The most generally recognized form of barotrauma is the presence of extra-alveolar air. A high airway pressure is an important factor in the pathophysiology of barotraumas [1]. The risk of excessive airway pressure is higher during manual ventilation of an intubated patient due to an absence of an air leak [2]. Such high pressures can injure the alveoli in some patients [3].

Transport can also pose a significant risk to mechanically ventilated critically ill patients. The changes in the patient's position can significantly affect the ETT depth. Patient movement during transport can cause the migration of an already marginally acceptable ETT position, which can lead to barotrauma.

Although many authors have reported barotrauma after positive pressure ventilation, there are few reports of barotrauma occurring after manual ventilation of intubated patients during transport. We report a case of bilateral pneumothorax, pneumopericardium, pneumo-mediastinum, pneumoretroperitoneum and subcutaneous emphysema following manual ventilation in a patient during transfer from ICU to the OR.

\section{Case Report}

A 74-year-old male patient receiving ventilatory support due to aspiration pneumonia was transferred to the OR from the ICU for an emergency operation of the sigmoid colon volvulus. In the ICU, the preoperative vital signs were blood pressure (BP) of 120/70 mmHg, heart rate (HR) of 70 beats/min, and

Received: February 26, 2010. Revised: 1st, March 11, 2010; 2nd, April 6, 2010. Accepted: April 20, 2010.

Corresponding author: Kyong Shil Im, M.D., Department of Anesthesiology and Pain Medicine, The Catholic University of Korea College of Medicine, Uijeongbu St. Mary's Hospital, 65-1,Geumo-dong, Uijeongbu 480-130, Korea. Tel: 82-31-820-3087, Fax: 82-31-821-8449, E-mail: idonga@catholic.ac.kr

(c) This is an open-access article distributed under the terms of the Creative Commons Attribution Non-Commercial License (http:// creativecommons.org/licenses/by-nc/3.0/), which permits unrestricted non-commercial use, distribution, and reproduction in any medium, provided the original work is properly cited. 
percutaneous oxygen saturation $\left(\mathrm{SpO}_{2}\right)$ of $98 \%$ on a fractional inspired oxygen concentration $\left(\mathrm{FiO}_{2}\right)$ 0.4. The preoperative chest $\mathrm{X}$-ray revealed diffuse ground glass opacity in middle lower lung suggesting aspiration pneumonia (Fig. 1). The patient had been ventilated mechanically using synchronized intermittent mandatory ventilation mode with a tidal volume of $450 \mathrm{ml}$ at a respiratory rate of 16 breaths/min, inspiratory : expiratory ratio of $1: 2$, positive end-expiratory pressure (PEEP) of $6 \mathrm{cmH}_{2} \mathrm{O}$, and $\mathrm{FiO}_{2}$ of 0.4 . The self respiratory rate was $18-21$ breaths/ min. Arterial blood gas analysis on a $\mathrm{FiO}_{2}$ of 0.4 revealed a $\mathrm{pH}$, $\mathrm{PO}_{2}, \mathrm{PCO}_{2}$ and $\mathrm{SpO}_{2}$ of 7.42, $125.9 \mathrm{mmHg}, 43 \mathrm{mmHg}$ and $98 \%$, respectively. The patient received manual ventilation with a self-inflated resuscitator bag (Ambu bag) during transport.

Upon entrance into the OR, he suffered cyanosis and showed a delirious mental state. The $\mathrm{SpO}_{2}$ was $64 \%$. Electrocardiogram revealed tachycardia (HR 130 beats $/ \mathrm{min}$ ) and the BP was 100/70 mmHg. The physical examination showed decreased breath sounds on the left. Manual ventilation was difficult and an adequate tidal volume was not delivered, even with considerable effort. Endobronchial intubation was suspected. The ETT was secured at $22 \mathrm{~cm}$ from the ETT tip and was found to be patent. It was then withdrawn slowly to $20 \mathrm{~cm}$. However, the breath sounds and $\mathrm{SpO}_{2}$ did not change. At that moment, subcutaneous emphysema with crepitus and hyperresonant percussion on the left abdomen was found. A pneumothorax was strongly suspected. Accordingly, rapid insertion of a 16 gauge angiocatheter into the left side of the chest was

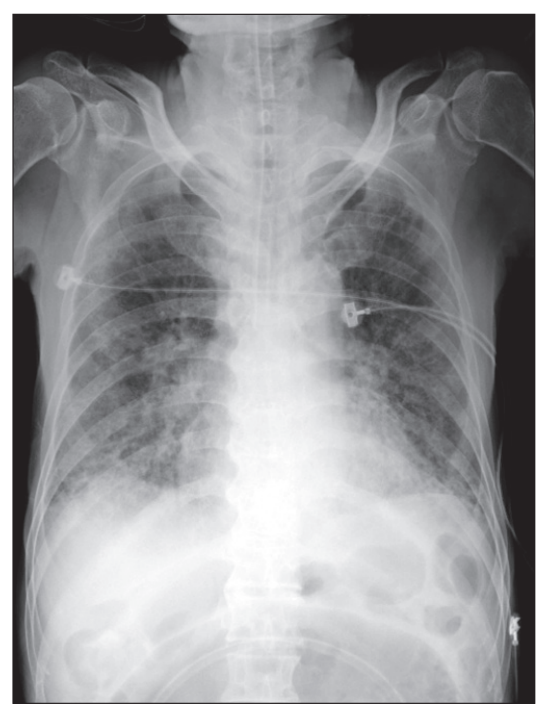

Fig. 1. Preoperative chest X-ray shows ill-defined patchy ground glass opacity in both middle lower lungs suggesting underlying aspiration pneumonia, considering the patient's history and minimal fibrotic scar in left apex. It also reveals endotracheal tube tip is placed in near the carina. performed and the air was released immediately. Immediately, a 28-French chest tube was inserted into the left side of the thorax. The cyanosis disappeared and vital signs were improved. The $\mathrm{SpO}_{2}$ increased but was below $90 \%$. A chest X-ray was taken, which revealed bilateral pneumothorax, pneumomediastinum, pneumopericardium, pneumoretroperitoneum, and subcutaneous emphysema (Fig. 2). A chest drain was also inserted into the right side of the thorax. The $\mathrm{SpO}_{2}$ increased to $100 \%$. The operation was postponed until the patient improved and transferred to the ICU. The sigmoidectomy was performed 10 days later. However, the patient expired because of an aggravation of pneumonia and residual pneumothorax on the 12th postoperative day.

\section{Discussion}

The most widely recognized form of barotrauma is the presence of extra-alveolar air. The most common mechanism for extra-alveolar gas formation is a disruption of the alveolar epithelium, leading to the entry of gas into the perivascular sheath. The gas then dissects within this sheath toward the mediastinum where it decompresses through other fascial planes producing the radiographic and clinical manifestations of pneumothorax, subcutaneous emphysema, subpleural air cysts, pneumomediastinum, pneumopericardium and pneumoperitoneum [4]. The risk of barotrauma is increased by excessive peak inspiratory pressure, high PEEP, high

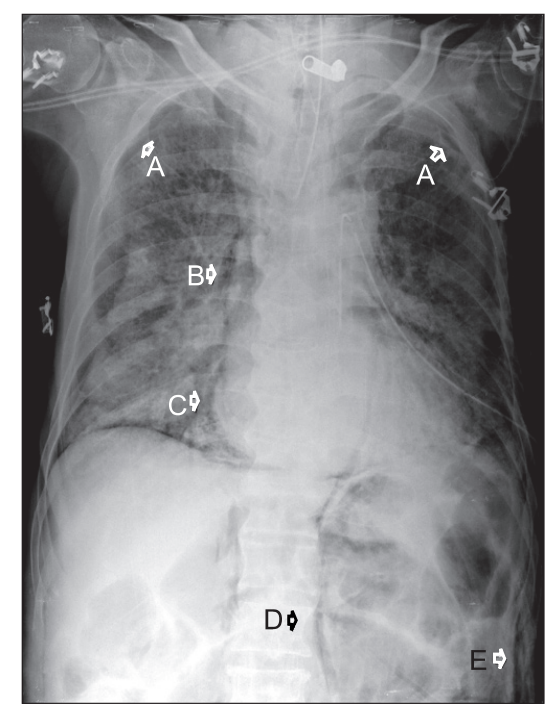

Fig. 2. Chest X-ray reveals bilateral pneumothorax (A) with a chest tube inserted on the left chest, pneumomediastium (B), pneumopericardium (C), pneumoretroperitoneum (D) and subcutaneous emphysema (E). Chest X-ray shows ill-defined patchy ground glass opacity in both middle lower lungs suggesting underlying aspiration pneumonia and minimal fibrotic scar in left apex. 
transalveolar pressure, alveolar overdistention and underlying lung injury [1]. The incidences of pneumothorax in patients receiving mechanical ventilation varies from 4 to $15 \%$ but might be significantly higher in patients with adult respiratory distress syndrome, status asthmaticus, and aspiration pneumonia [5]. In the present case, underlying aspiration pneumonia might have predisposed the patient to the development of barotrauma.

Manual ventilation is frequently required during intrahospital transport. Because clinicians are relatively unconstrained in manual ventilation, the ventilatory patterns can differ significantly among clinicians, and may be inappropriate and deleterious in certain circumstances [6]. A high airway pressure with excessive or inappropriate manual ventilation can cause a pneumothorax and/or other pulmonary air leak problems. In this case, it is likely that the inappropriate manual ventilation induced a high airway pressure or alveolar overdistension, resulting in these complications. The accuracy and stability of manual ventilation can contribute to the quality of ventilation and prevent barotrauma. Accurate and stable manual ventilation can be provided by appropriate training and when a manometer is used in conjunction with the bag. Therefore, physicians should train with a test lung in order to help them realize the ventilation patterns they may generate during manual ventilation.

In this case, the possibility of a preoperative pneumothorax that might have developed in the ICU and remained undetected in the preoperative chest X-ray could not be excluded. It is difficult to make a radiographic diagnosis of a pneumothorax on a portable chest X-ray film taken in the ICU [5]. The traditional radiographic hallmark of a pneumothorax may not manifest in portable supine films of patients with a pneumothorax. In addition, concurrent lung disease might lead to different distributions of free air in the pleural space than in patients with relatively normal lungs [7]. Despite the supine chest $\mathrm{x}$-ray film being reviewed carefully, a pneumothorax can be missed without using other imaging modalities or techniques. Several studies reported that computed tomography (CT) scans of the chest may be more sensitive in detecting a pneumothorax in ICU patients than portable chest radiographs [8-10]. However, it is not always practical or safe to transport critically ill patients for a CT scan to exclude a latent pneumothorax, particularly when the patient is hemodynamically unstable. A preoperative CT scan was not performed in this case.

Manual ventilation can shift the secured ETT within the trachea. Therefore, the migration of an already marginally acceptable ETT position by manual ventilation during transport is believed to be the cause of these complications. The ETT tip was sited near the carina in the preoperative chest X-ray. During transport, migration of the ETT tip to the endobronchial position might lead to a high airway pressure that can result in barotrauma. In the present case, the deep placement of an ETT was not detected in the ICU. Auscultation is not sensitive in detecting ETT tip placement in the main bronchus. Sugiyama et al. [11] demonstrated normal breath sounds with endobronchial depths of $1.5-2.0 \mathrm{~cm}$, and did not detect the disappearance of breath sounds until a depth of $3.2 \mathrm{~cm}$. Ezri et al. [12] reported there were no signs of a change in $\mathrm{SpO}_{2}$ in the cases of endobronchial intubation.

Tension pneumothorax is believed to occur when the communication between the lung parenchyma and pleural space acts as a one-way valve, allowing air to enter the pleural cavity during inspiration but trapping air during expiration. The development of tension is dependent on the pressure gradient between the intrapleural pressure and alveolar pressure. Ventilation will increase the gas flow through the pleural defect, allowing more air to pass per unit time and a more rapid increase in intrapleural pressure with earlier mechanical compressive effects and rapid progress to cardiorespiratory collapse. Therefore, tension pneumothorax develops more commonly in patients on mechanical ventilation than in spontaneously breathing patients. Moreover, ventilated patients with tension pneumothorax deteriorate much more rapidly, and sudden cardiac arrest is more likely [13]. The immediate treatment for tension pneumothorax is needle decompression, which is only a temporary measure until tube thoracostomy can be performed. The definitive treatment of tension pneumothorax is tube thoracostomy.

Strange [14] reported that a clinical diagnosis could be made immediately in $61 \%$ of patients with a tension pneumothorax, of which $7 \%$ had died. A mortality of $31 \%$ was recorded in a group of patients in which clinical signs preceded the diagnosis by 30 minutes to 8 hours. Tension pneumothorax occurred more frequently in patients with an initially misdiagnosed pneumothorax than in patients with a correctly diagnosed. The risk factors predisposing a patient to a misdiagnosis of a pneumothorax in the ICU include mechanical ventilation required at the time of development of a pneumothorax, atypical radiographic location of the pneumothorax, altered mental status exhibited at the time of pneumothorax presentation and the development of a pneumothorax after peak physician staffing hours. In this case, the development of a pneumothorax was not recognized during transport. The intubation state and transport without a specially trained transport team might have contributed to the failure to make an early diagnosis of a pneumothorax during transfer. Knowledge of the factors predisposing the patient to this problem should allow a higher index of suspicion for a diagnosis of a pneumothorax in critically ill patients and possibly improve the early recognition of a pneumothorax.

In conclusion, inappropriate manual ventilation, deep 
placement of an ETT and aggravation of an undetected preoperative pneumothorax are likely to have contributed to these complications. Accurate and stable manual ventilation, appropriate placement and thorough management of an ETT, as well as an immediate diagnosis and correct treatment can prevent this outcome. In addition, the ability of continual monitoring and appropriate patient care during transport is essential for preventing transport-related morbidity and mortality.

\section{References}

1. Gammon RB, Shin MS, Buchalter SE. Pulmonary barotrauma in mechanical ventilation. Patterns and risk factors. Chest 1992; 102: 568-72.

2. Ricard JD. Manual ventilation and risk of barotrauma: primum non nocere. Respir Care 2005; 50: 338-9.

3. Turki M, Young MP, Wagers SS, Bates JH. Peak pressures during manual ventilation. Respir Care 2005; 50: 340-4.

4. Chen YL, Chen CY, Cheng JK. Delayed tension pneumothorax during surgery. J Chin Med Assoc 2005; 68: 491-4.

5. Tocino I, Westcott JL. Barotrauma. Radiol Clin North Am 1996; 34: 59-81.
6. McCarren B, Chow CM. Manual hyperinflation: a description of the technique. Aust J Physiother 1996; 42: 203-8.

7. Chiles C, Ravin CE. Radiographic recognition of pneumothorax in the intensive care unit. Crit Care Med 1986; 14: 677-80.

8. Wall SD, Federle MP, Jeffrey RB, Brett CM. CT diagnosis of unsuspected pneumothorax after blunt abdominal trauma. AJR Am J Roentgenol 1983; 141: 919-21.

9. Holmes JF, Brant WE, Bogren HG, London KL, Kuppermann N. Prevalence and importance of pneumothoraces visualized on abdominal computed tomographic scan in children with blunt trauma. J Trauma 2001; 50: 516-20.

10. Rubinowitz AN, Siegel MD, Tocino I. Thoracic imaging in the ICU. Crit Care Clin 2007; 23: 539-73.

11. Sugiyama K, Yokoyama K, Satoh K, Nishihara M, Yoshitomi T. Does the Murphy eye reduce the reliability of chest auscultation in detecting endobronchial intubation? Anesth Analg 1999; 88: 1380-3.

12. Ezri T, Khazin V, Szmuk P, Medalion B, Shechter P, Priel I, et al. Use of the Rapiscope vs chest auscultation for detection of accidental bronchial intubation in non-obese patients undergoing laparoscopic cholecystectomy. J Clin Anesth 2006; 18: 118-23.

13. Leigh-Smith S, Harris T. Tension pneumothorax--time for a rethink? Emerg Med J 2005; 22: 8-16.

14. Strange C. Pleural complications in the intensive care unit. Clin Chest Med 1999; 20: 317-27. 